\title{
Dominantly Inherited Ataxias: Lessons Learned from Machado-Joseph Disease/ Spinocerebellar Ataxia Type 3
}

\author{
Henry L. Paulson, M.D., Ph.D. ${ }^{1}$
}

To date, nearly 30 distinct genetic forms of dominantly inherited ataxia are known to exist. Of these, Machado-Joseph disease (MJD), also known as spinocerebellar ataxia type 3 (SCA3), is perhaps the most common in many regions of the world including the United States. This article discusses MJD/SCA3 as a paradigm example of the dominant ataxias, which are collectively known as the spinocerebellar ataxias. Using MJD/SCA3 as a starting point, the article reviews common clinical and genetic features of the SCAs and highlights new insights into molecular mechanisms, especially of the SCAs caused by polyglutamine expansion. Also discussed are current and future therapeutic opportunities for MJD/SCA3 in particular, many of which have relevance to other SCAs.

KEYWORDS: ataxia, polyglutamine, cerebellum, neurogenetics

Machado-Joseph disease (MJD), also known as spinocerebellar ataxia type 3 (SCA3), is one of a growing number of genetically defined, dominantly inherited ataxias. Collectively, the dominant ataxias are known as the spinocerebellar ataxias. SCAs are numbered by the order in which their genetic loci were discovered. MJD/ SCA3 was the third to be identified, hence its designation as SCA3. At least 28 distinct genetic forms of SCA have been identified, with many more likely to be found (Table 1). This huge number can be daunting, if not downright depressing, to a neurologist. It is impossible to keep this many separate genetic forms of disease distinct in one's memory, especially if one also hopes to reserve memory space for the equally impressive number of genetic forms of hereditary recessive ataxias, spastic paraplegias, and neuropathies.

$\mathrm{MJD} / \mathrm{SCA} 3$ is a relatively rare neurodegenerative disorder. ${ }^{1}$ Practically speaking, such a rare disease barely merits its own article in this clinically oriented volume. Accordingly, MJD/SCA3 is featured here as a paradigm example of the SCAs as a whole. The goal is not to provide an exhaustive overview of all the clinical and molecular features of this disease and of the SCAs as a class. Rather, MJD/SCA3 is discussed as a prototypical SCA with which to emphasize key, shared features among the SCAs.

Why use MJD/SCA3 as a paradigm exampleother than the fact that the author has a special research interest in this disorder? First, and perhaps most important, MJD/SCA3 may be the most common SCA in the world. Studies in numerous regions of the world have shown that MJD/SCA3 comprises between 15 and 45\% of all SCAs in many different countries and ethnic populations. In the ethnic melting pot of the United States, MJD/SCA3 joins SCA2 and SCA6 as the three most common ataxias. A second reason is that perhaps more than any other SCA, MJD/SCA3 manifests with a remarkably broad range of phenotypes. Because phenotypic heterogeneity is common to many SCAs, a discussion of the varying clinical features in MJD/SCA3

\footnotetext{
${ }^{1}$ Department of Neurology, University of Iowa College of Medicine, Iowa City, Iowa.

Address for correspondence and reprint requests: Henry L. Paulson, M.D., Ph.D., Department of Neurology, University of Iowa College of Medicine, 3160 Med Labs, Iowa City, IA 52242.
}

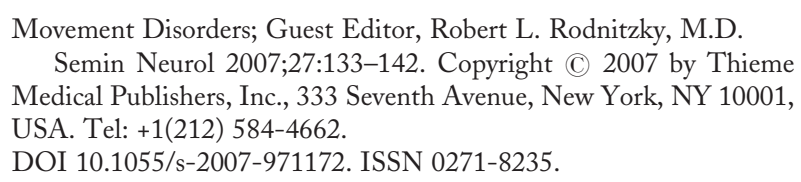

DOI 10.1055/s-2007-971172. ISSN 0271-8235. 
Table 1 Currently Known Spinocerebellar Ataxias

\begin{tabular}{|c|c|c|c|}
\hline Disease & Locus & Gene (product) & Type of Mutation \\
\hline SCA1 & $6 p 22.3$ & SCA1 (ataxin-1) & CAG/polyQ expansion \\
\hline SCA2 & $12 q 24.12$ & SCA2 (ataxin-2) & CAG/polyQ expansion \\
\hline SCA3 (MJD) & $14 q 32.21$ & MJD1 (ataxin-3 or MJDp) & CAG/polyQ expansion \\
\hline SCA4 & $16 q 22.1$ & Unknown & Unknown \\
\hline SCA5 & $11 p 11-q 11$ & $\beta$ III Spectrin & Nonrepeat mutations \\
\hline SCA6 & $19 p 13.2$ & CACNA1 & CAG/polyQ expansion \\
\hline SCA7 & 3p14.1 & Ataxin-7 & CAG/polyQ expansion \\
\hline SCA8 & $13 q 21.33$ & SCA8 & CTG/CAG expansion \\
\hline SCA10 & $22 q 13$ & SCA10 & ATTCT expansion \\
\hline SCA11 & $15 q 14-21.3$ & Unknown & Unknown \\
\hline SCA12 & $5 q 32$ & PPP2R2B & CAG expansion \\
\hline$\underline{\mathrm{SCA} 13}$ & 19q13.3-13.4 & $\mathrm{KCNC3}$ & Nonrepeat mutations \\
\hline$\underline{\text { SCA14 }}$ & 19q13.4-q ter & PKRCG & Nonrepeat mutations \\
\hline SCA15 & $3 p t e r-q 24.2$ & Unknown & Unknown \\
\hline SCA16 & $8 q 23-24.1$ & Unknown & Unknown \\
\hline SCA17 & $6 q 27$ & TBP & CAG/polyQ expansion \\
\hline SCA18 & $7 q 31-32$ & Unknown & Unknown \\
\hline SCA19 & $1 p 21-q 21$ & Unknown & Unknown \\
\hline SCA21 & 7p21.3-15.1 & Unknown & Unknown \\
\hline SCA22 & $1 p 210 q 23$ & Unknown & Unknown \\
\hline SCA23 & 20p13-12.2 & Unknown & Unknown \\
\hline SCA24 & $1 \mathrm{p} 36$ & Unknown & Unknown \\
\hline SCA25 & $2 p 21-p 15$ & Unknown & Unknown \\
\hline SCA26 & 19p13.3 & Unknown & Unknown \\
\hline$\underline{\mathrm{SCA} 27}$ & 13 & FGF14 & Nonrepeat mutations \\
\hline SCA28 & $18 p 11.22-q 11.2$ & Unknown & Unknown \\
\hline
\end{tabular}

Note that of those diseases in which mutations have been identified, many are repeat-expansion diseases (indicated with bold type). In addition, recent discoveries have revealed SCAs that are caused by nonrepeat mutations (underlined).

SCA, spinocerebellar ataxia; polyQ, polyglutamine; MJD, Machado-Joseph disease.

serves as an introduction to the phenotypic heterogeneity seen among SCAs as a whole. A third reason is that new insights into the function of the disease gene in MJD/ SCA3 have begun to shed light on molecular mechanisms for an entire class of related genetic disorders, the so-called expanded polyglutamine (polyQ) diseases. Because many SCAs have the same type of dynamic repeat mutation as MJD/SCA3, mechanistic insights from any one of these diseases may lead to rational therapies for the entire class of diseases.

The following sections discuss key facts or issues regarding $\mathrm{MJD} / \mathrm{SCA} 3$ that also pertain to SCAs more generally. The topics range from clinical to therapeutic to mechanistic. In choosing references for this article, I have emphasized more recent sources; however, several important, earlier articles in the field are also cited.

\section{CLINICALLY, SPINOCEREBELLAR ATAXIAS OFTEN INVOLVE MUCH MORE THAN PROGRESSIVE ATAXIA}

Just as patients with parkinsonism may prove to have one of several Parkinson-plus disorders, so, too, those with SCAs may have "ataxia-plus" syndromes. Although pro- gressive gait and limb ataxia is a core clinical feature of $\mathrm{SCAs}$ - in most cases the predominant, disabling feature of disease - the ataxia is usually accompanied by signs and symptoms that reflect damage beyond the cerebellum and its afferent and efferent pathways. Depending on the particular SCA, these affected regions can include the brainstem, basal ganglia, thalamus, spinal cord, peripheral nervous system, and, less often, cerebral cortex.

An older classification of dominantly inherited ataxias, the autosomal-dominant cerebellar ataxia (ADCA) classification put forth by Anita Harding, placed most of these hereditary ataxia-plus syndromes into the ADCA type 1 group. In ADCA type 1 , ataxia is accompanied by significant neurological signs referable to brainstem and/or spinal cord involvement. MJD/SCA3 is squarely in the ADCA type 1 camp, as are most of the common SCAs. ADCA type 2 represents a much less common ataxia-plus syndrome that, by definition, includes retinal degeneration. SCA7 currently is the only identified SCA in which retinal involvement leading to blindness is a hallmark clinical feature. Thus, SCA7 is the only known SCA that falls into the ADCA type 2 category. ADCA type 3 represents a "pure" cerebellar ataxia syndrome, with or without vestibular symptoms. 
ADCA type 3 is represented by SCA6, which is an exception to the more common ataxia-plus presentation of most SCAs. Other hereditary pure cerebellar ataxias exist, but the genes have not yet been identified.

The ataxia-plus syndrome characteristic of SCAs is clearly the rule for MJD/SCA3. Most patients with MJD/SCA3 first develop signs of disease in early-tomid adult years. Often, symptoms start with unsteadiness upon turning, followed by overt gait imbalance. Within a few years, this progresses to wide-based gait ataxia accompanied by subtle limb incoordination and a cerebellar dysarthria with scanning speech. Eventually, gait and limb ataxia becomes so severe that the patient needs to use gait-assist devices, culminating in the use of a wheelchair. As signs of cerebellar involvement progress, many patients also develop oculomotor disturbance; bulging, staring eyes; hyperreflexia; lower limb spasticity; and parkinsonian features. These neurological signs clearly do not reflect cerebellar involvement; rather, they correspond to neuropathological changes occurring in the basal ganglia, brainstem, spinal cord, and peripheral nervous system. Recent studies have shown, for example, that degeneration in MJD/SCA3 is particularly severe in many brainstem nuclei, much more so than in the molecular and Purkinje cell layers of the cerebellum..$^{2-4}$ It is perhaps telling that the most frequently noted magnetic resonance imaging abnormality in MJD/ SCA3 is enlargement of the fourth ventricle, which likely reflects a combination of atrophy in the deep cerebellar nuclei and the brainstem. ${ }^{5}$

Extracerebellar involvement also applies to most other SCAs. Among the most common SCAs, SCAs 1, 2 , and 7 also typically display noncerebellar features that often are as disabling as the ataxia. The one common pure cerebellar ataxia is SCA6, in which all of the clinical features can be ascribed to cerebellar degeneration. Consistent with this, SCA6 shows selective cerebellar atrophy without brainstem atrophy, unlike MJD/SCA3 and many other SCAs.

Relatively spared in most SCAs are the brain's cerebral hemispheres. Some cognitive problems are observed in most SCAs, including in MJD/SCA $3,{ }^{6}$ but very little cerebral atrophy occurs. An exception is SCA17, in which patients have severe cognitive problems associated with global cerebral atrophy.

It is important to recognize that the ataxia-plus presentation and disease course described here are typical for "classic," midlife onset MJD/SCA3, which is the most common presentation of MJD/SCA3. More rarely, individuals with earlier onset MJD/SCA3 (occurring in their teens or early 20s) may suffer more from dystonia and bradykinesia than from ataxia. In addition, the latest onset patients, whose disease can begin after age 50, may have as much difficulty from neuropathy as from ataxia. The following sections describe further the reasons for these age-dependent clinical differences.

\section{CLINICAL SPECTRUM OF MACHADO- JOSEPH DISEASE AND OTHER SPINOCEREBELLAR ATAXIAS VARIES WIDELY}

Even within the same family, persons affected with MJD/SCA3 can have widely varying clinical signs and symptoms. Indeed, it is telling that the disease has two names. When clinicians and scientists began defining an inherited ataxia in French and German families, which came to be known as SCA3, the disease features seemed distinct enough from those experienced by affected families with MJD to assume that it was a separate disease. MJD had already been described and mapped to chromosome 14. Only when the actual MJD mutation was discovered in $1994^{7}$ did these same clinicians discover, to their surprise, that $\mathrm{SCA} 3$ was in fact the same disease as MJD. ${ }^{8}$ Hence, the designation as MJD/SCA3, although the official name given by the Human Genome Organization (HUGO) Nomenclature Committee is MJD.

Clinical features vary so much in MJD/SCA3 that clinicians have even taken the trouble to classify various subtypes of disease. ${ }^{8-12}$ Type $1 \mathrm{MJD} / \mathrm{SCA} 3$ begins the earliest, often before age 20 years. It is characterized by rigidity, spasticity, and bradykinesia, with ataxia being less of a problem (although it is clearly present). Type 2 $\mathrm{MJD} / \mathrm{SCA} 3$ is the most common type, with symptoms typically beginning during mid adult years. The type 2 presentation constitutes the classic ataxia-plus syndrome. Type 3 is a later onset form of disease in which peripheral nerve and lower motor neuron involvement leads to lower limb amyotrophy and areflexia, together with ataxia. Some have even designated a fourth class, type 4 disease, which is characterized by parkinsonism that is often responsive to levodopa therapy. ${ }^{13-15}$

Why is there such wide variability in clinical features? Simply put, because the disease-causing mutation is a dynamic repeat expansion. In MJD/SCA3 and at least five other SCAs, the mutation is an unstable, expanded CAG repeat that encodes a tract of glutamine residues in the disease protein. ${ }^{16}$ In general, for most dynamic repeat mutations and certainly for the CAG repeat diseases, the larger the expansion the more severe the mutation: Larger expansions tend to cause disease earlier in life with a wider range of neuropathological involvement. Conversely, smaller expansions cause later onset disease that in rare cases can be compatible with a normal life span. Consistent with this, the various types of MJD/SCA3 can be mapped to different size repeats: Type 1 disease tends to be caused by the largest repeats, type 2 by midrange expansions, and type 3 by the smallest expansions. The so-called type 4 disease with parkinsonism does not easily conform to a particular repeat range; most likely, other genetic and environmental factors help to specify the presence or absence of parkinsonism in MJD/ SCA3. Although repeat size is not the only aspect 


\section{Normal allele}

\section{DNA: ...nnCAGCAGCAGnn...}

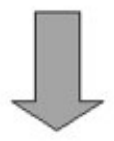

\section{protein:}

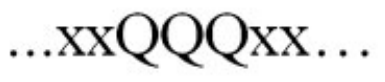

\section{Disease allele}

...nnCAGCAGCAG

CAGCAGCAGCAG

CAGCAGCAGnn...
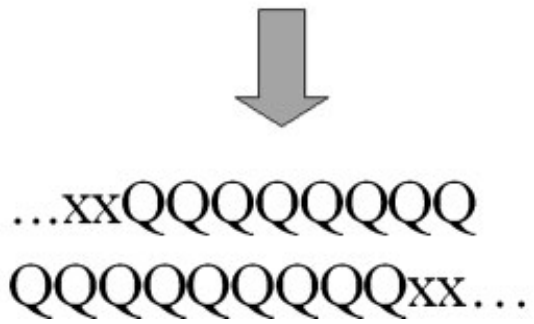

Figure 1 CAG repeat expansions encode abnormally long tracts of glutamine. Both normal and disease alleles contain repeats that encode glutamine repeats, shown here as $\mathrm{Q}$, which is the single-letter amino acid code for glutamine. Spinocerebellar ataxias (SCAs) known to be caused by polyglutamine-encoding CAG expansions are SCAs 1, 2, 3 (MJD), 6, 7, and 17.

that specifies age of onset and disease features, it clearly is the most important factor.

This wide-ranging clinical variability is also seen in the other SCAs caused by dynamic repeat expansions. As in MJD/SCA3, variability in repeat length underlies the clinical heterogeneity observed in each of the expanded repeat SCAs. In addition to SCA3, SCAs $1,2,6,7$, and 17 are all caused by $\mathrm{CAG}$ repeat expansions. Each of these diseases shows a similar repeat length-disease correlation to $\mathrm{MJD} / \mathrm{SCA} 3$ - a strong inverse correlation between repeat length and age of disease symptom onset. Longer repeats cause earlier onset disease with a broader range of neuropathological involvement.

In addition to the protein-coding CAG repeat diseases, several other SCAs are caused by expanded repeat sequences. For example, SCA10 is due to an extremely large pentanucleotide expansion in an intron, and SCA 12 is caused by a rather small, noncoding CAG repeat expansion in the $5^{\prime}$ region of the disease gene. A third expanded repeat SCA, SCA8, remains a bit confusing as the CTG expansion is not fully penetrant. Moreover, the SCA8 expansion appears to be transcribed in both directions, producing a CUG repeat in one direction (which might act through a dominant RNA mechanism) and a CAG repeat in the other direction (encoding an expanded polyQ protein). ${ }^{17}$

\section{DISCOVERY OF THE UNDERLYING GENETICS HAS SHED LIGHT ON CONFUSING CLINICAL FEATURES}

The 1990s was a landmark decade for neurogenetics. It was during the early 1990s that dynamic repeat expansions were discovered. In quick succession, spinobulbar muscular atrophy (SBMA) (also known as Kennedy's disease), fragile $\mathrm{X}$ mental retardation syndrome, and myotonic dystrophy type 1 were found to be caused by unstable DNA repeat expansions. Soon thereafter, SCA1 and Huntington's disease (HD) were discovered to be caused by CAG repeat expansions that, as in SBMA, encoded an expanded tract of the amino acid glutamine in the respective disease proteins. This led to the idea of a class of neurodegenerative diseases in which similar mutations encode expanded polyQ that is toxic to neurons (Fig. 1).

In 1994, MJD/SCA3 was found to belong to this same class of polyQ disorders. ${ }^{7}$ A CAG repeat expansion in the MJD1 gene encodes an expanded polyQ tract in the disease protein, known as ataxin-3 or MJDp. Since this discovery, we have learned that at least nine diseases, and possibly more, are due to polyQ-coding CAG repeat expansions. ${ }^{16}$

The discovery of dynamic repeat mutations helped to clarify many mysteries about MJD and other disorders. For example, the startling range of clinical variability that can be seen in MJD/SCA3, even within a given family, is explained by the presence of differing repeat sizes in different affected individuals.

A second mystery solved by the discovery of the underlying genetics is the phenomenon known as "anticipation." Some clinicians had long noticed that certain inherited diseases tended to worsen from generation to generation-in a word, anticipation. Although anticipation does occur in MJD/SCA3, it is more impressive and more common in many other dynamic repeat expansion disorders. Among the polyQ diseases, anticipation is most clearly seen in SCA2, SCA7, and HD. Before the discovery of expanded repeats, some discounted the notion of anticipation as biologically implausible. But once the mutations were discovered to be unstable expansions, it quickly became clear that 
anticipation is a genuine biological phenomenon in certain disorders, where it is explained by the unusual behavior of these mutations. Anticipation reflects the tendency of expanded repeats to get larger when transmitted from one generation to the next. This intergenerational tendency for further expansion, coupled with the fact that longer repeats tend to cause more severe disease beginning earlier in life, provides a molecular explanation to anticipation.

\section{AS WE LEARN MORE ABOUT THE UNDERLYING GENETICS OF MACHADO- JOSEPH DISEASE/SPINOCEREBELLAR ATAXIA TYPE 3, THE PHENOTYPIC RANGE BROADENS}

Soon after the CAG repeat expansion in MJD/SCA3 was discovered, it seemed evident that a gap existed between the normal repeat range (roughly 12-40) and the disease repeat range $(\sim 60-84)$. This set MJD/SCA3 apart from the other identified CAG repeat SCAs in which there is relatively little separation between normal and expanded repeat sizes. This rather wide gap at the MJD locus suggested that there was not an intermediate range of repeats in which expansions are incompletely penetrant as is seen, for example, in SCA2. As time has passed, however, cases have surfaced in which people do carry intermediate size MJD repeats $(>42$ but $<60) .{ }^{18-20}$ In some of these cases, carriers of intermediate repeats develop clear-cut progressive ataxia, indicating that the intermediate repeat is in fact a fully manifesting mutation. For example, a mother-daughter pair presented in my ataxia clinic, both of whom had subtle dysarthria, gait ataxia, mild intention tremor, and dysdiadochokinesia. Their MJD repeat lengths were 54 and 52, respectively. Although the official genetic testing report, obtained through a commercial laboratory, called these "repeats of indeterminate length," the fact that both women showed classic features of an ataxia syndrome indicated these repeats were "determinant" repeats-disease-causing expansions that, although not officially falling within the classically defined pathogenic range, caused progressive ataxia in these two family members. This scenario is admittedly rare, as relatively few individuals have large normal repeats or intermediate size repeats at the $M J D$ locus. ${ }^{21}$ But it does illustrate that, over time, our view of what is a disease versus a nondisease repeat length can change based on new findings. For any genetic disorder, it is important that ongoing clinical assessment continues to incorporate new advances in our knowledge of the underlying genetics. This is certainly true for MJD/SCA3.

A second recent discovery is that individuals with such intermediate size MJD expansions ( $\sim 2$ to $\sim 58)$ may develop isolated restless legs syndrome (RLS) without features of progressive ataxia. RLS is also fairly common in full blown MJD/SCA3 and can respond well to dopamine agonists or levodopa. I should emphasize that intermediate size MJD repeats are uncommon in the general population, so they are not a frequent cause of sporadic RLS in any patient population. ${ }^{22}$ However, in those with a family history of ataxia, the presence of RLS may reflect subtle manifestations of MJD/SCA3. The presence of RLS in MJD/SCA3 is intriguing, especially when one considers that nigral neurons and peripheral neurons are both susceptible to degeneration in this disorder.

\section{A CENTRAL PARADOX OF POLYGLUTAMINE DISEASES: SAME MUTATION RESULTS IN DIFFERENT DISEASES}

$\mathrm{MJD} / \mathrm{SCA} 3$ is one of at least nine disorders caused by polyQ-encoding CAG repeat expansions. Current views about polyQ pathogenesis largely center around protein misfolding and the problems that ensue from such misfolding. ${ }^{23-27}$ Models of pathogenesis include the formation of toxic oligomers of the disease protein as well as aberrant protein-protein interactions that perturb specific cellular functions as diverse as gene expression, subcellular localization, axonal trafficking, protein homeostasis, and protein clearance.

Despite this presumably shared pathogenic mechanism, however, the nine known polyQ diseases are distinct disorders. No neurologist would mistake the ataxia of MJD/SCA3 for the chorea of $\mathrm{HD}$ or the lower motor neuron disease of SBMA. Even among the polyQ $\mathrm{SCAs}$, the clinical features prominent in a given SCA provide powerful clues to distinguish it from others. For example, retinal degeneration is the domain of SCA7; bulging, staring eyes are most commonly seen in MJD/ $\mathrm{SCA} 3$; parkinsonism is seen in SCA2 and MJD/SCA3; and severe cognitive dysfunction occurs in SCA17.

Why do these diseases differ despite their common mutation? In two words, protein context. ${ }^{28}$ Each mutation occurs in an otherwise completely unrelated protein. The various proteins differ in size, amino acid sequence, and, most importantly, cellular function(s). Moreover, the location within the protein of the polyQ expansion differs for each of the proteins, from very near the amino terminus (in the $\mathrm{HD}$ protein, huntingtin) to near the $\mathrm{C}$ terminus (in the MJD/SCA3 protein, ataxin-3).

Although it is clear that expanded polyQ is intrinsically neurotoxic, it is equally clear that expanded polyQ by itself cannot replicate all of the disease features of any given polyQ disease in cellular and animal models. This is perhaps clearest in HD, where the first reported transgenic mouse model expressed only the first exon of this 68-exon gene (the first exon encodes the "start" methionine for the protein and very little else except for the polyQ expansion). This mouse, the well-studied "R6/2" mouse, develops a progressive neurodegenerative phenotype accompanied by extensive aggregation of the 

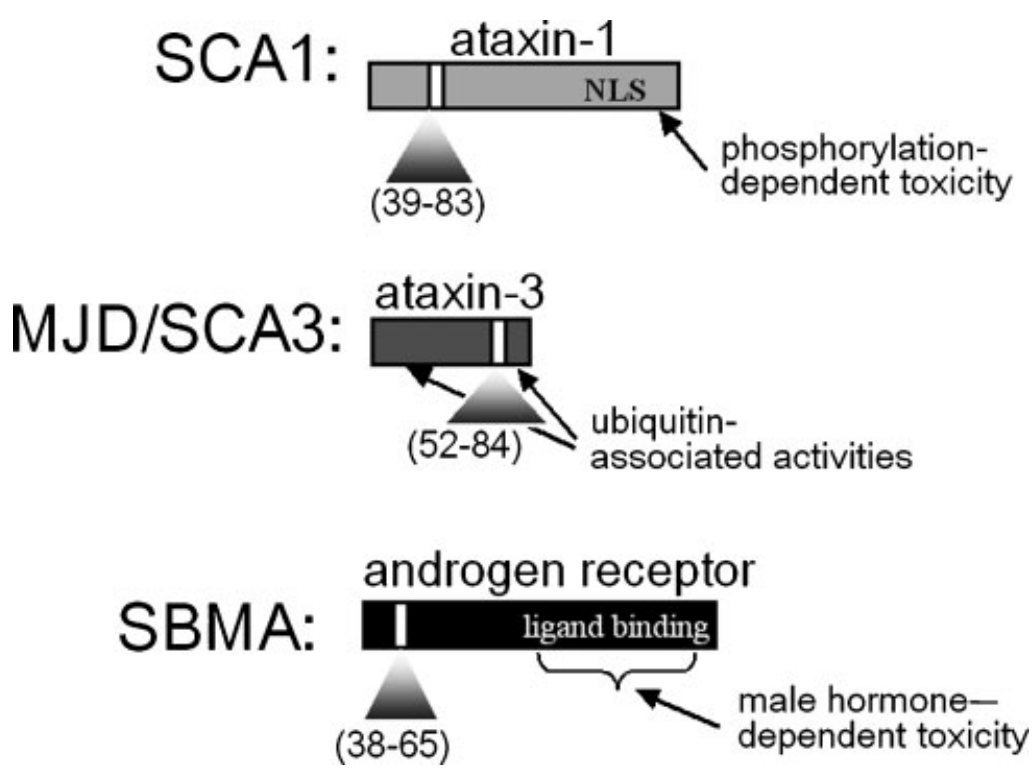

Figure 2 Protein context influences toxicity in polyglutamine (polyQ) diseases. Shown are three disease proteins in which regions far removed from the polyQ domain are known to influence toxicity of the disease protein. Arrows point to regions of the proteins in which phosphorylation (ataxin-1 in SCA1), hormone binding (androgen receptor in SBMA), and ubiquitin-related activities (ataxin-3 in MJD/ SCA3) modulate toxicity of the disease protein in animal models. SCA, spinocerebellar ataxia; NLS, nuclear localization signal; MJD, Machado-Joseph disease; SBMA, spinobulbar muscular atrophy.

polyQ-containing protein encoded by the transgene. This model does not, however, recapitulate the selective striatal degeneration that occurs in humans with HD. Perhaps it is better to think of it as a generic model of expanded polyQ degeneration rather than as an accurate model of huntingtin-mediated degeneration. HD mouse models expressing the full disease protein do a better job of recapitulating some of the HD-specific disease features, with the trade-off that the disease manifestations are milder and slower to develop in the mouse. Similarly, $\mathrm{MJD} / \mathrm{SCA} 3$ is modeled more accurately in transgenic flies or mice expressing full-length ataxin-3 rather than a polyQ-containing fragment of the disease protein. Again, the full-length ataxin-3 protein $^{29-31}$ causes a milder disease phenotype than a polyQ fragment derived from the disease protein. ${ }^{32,33}$

In general, regions outside the expanded polyQ tract modulate the toxicity of individual polyQ disease proteins. ${ }^{28} \mathrm{~A}$ few good examples of this are shown in Fig. 2. For example, toxicity of the SCA1 disease protein, ataxin-1, requires phosphorylation of a serine residue far removed from the actual disease mutation. When this serine is mutated so that it no longer can be phosphorylated, the disease protein does not cause toxicity in mice or in flies. This finding has led researchers to propose a model of SCA1 disease pathogenesis in which the mutant protein abnormally accumulates through a phosphorylation-dependent interaction with the 14-3-3 protein. A second compelling example of the importance of protein context is provided by the motor neuron disease SBMA. In this disorder, the toxicity of the disease protein, the androgen receptor (AR), depends on the presence of male hormones. Men with the mutation develop disease; women with the mutation essentially do not. Moreover, castrated male transgenic mice do not develop disease, whereas female mice treated with male hormones do. The binding site for androgens in the AR is far removed from the polyQ mutation, yet androgen binding is essential to disease pathogenesis.

The importance of protein context is very clear in $\mathrm{MJD} / \mathrm{SCA} 3$. The disease protein is known as ataxin-3 or MJDp. Whereas a carboxy-terminal fragment of expanded (pathogenic) ataxin-3 displays widespread toxicity in animal models, the full-length disease protein behaves very differently, being only mildly toxic. Mouse and fruit fly models expressing the full-length disease protein show a much milder, more selective neurodegeneration than does a polyQ-containing C-terminal fragment of the protein. Intriguingly, nonexpanded ataxin-3 can actually suppress the toxicity of other polyQ disease proteins in the fly. Ataxin-3 is unique among polyQ proteins; it is the only one that is also an intrinsic suppressor of polyQ toxicity. Mutant ataxin-3 appears to have a suppressor activity that counters its own polyQ toxicity.

This remarkable suppressor activity of ataxin-3 is linked to its involvement in ubiquitin-dependent protein degradation pathways. Ataxin-3 is a ubiquitin-binding protein that both binds and cleaves specific types of large ubiquitin chains. ${ }^{34-39}$ When its enzymatic activity is engineered to be inactivated, ataxin-3 no longer can suppress toxicity by other polyQ proteins and becomes somewhat toxic by itself. Ataxin-3 has also been shown to facilitate the transport of abnormal proteins to the degradation machinery of the cell. ${ }^{40}$ So much has been learned about ataxin-3 in the past few years, including new insights into its physical structure, ${ }^{38,39,41}$ that we can expect continued 
understanding of how it normally functions and how it fails to function properly in the disease state.

The unique protein context of each disease protein also may help explain the selective neuronal toxicity seen in these disorders. In general, each of the disease proteins is widely expressed in the brain, yet only certain brain regions are prone to degenerate. The affected brain area is perhaps most widespread in SCA17, followed by $\mathrm{MJD} / \mathrm{SCA} 3$, and is most restricted in SCA6. Most likely, the particular functions of each disease protein and the unique array of protein partners with which each disease protein interacts greatly influence which neurons will be adversely affected.

\section{DESPITE MAJOR ADVANCES, WE STILL LACK PREVENTIVE THERAPY}

All of the polyQ ataxias, including MJD/SCA3, are ultimately fatal disorders for which we have no preventive therapy. Patients typically die 15 to 30 years after the onset of symptoms from complications of swallowing dysfunction, immobility, and poor nutritional status. An exception to this general rule may be the pure cerebellar ataxias, of which SCA6 is the most common. Individuals with SCA6, particularly those with later onset symptoms, may even live a normal life span.

Many scientists suspect that protein misfolding lies at the heart of disease pathogenesis. Based on this view, approaches that boost molecular chaperone function in neurons to facilitate protein folding could slow disease. ${ }^{42}$ Many studies in cell and animal models support the idea that enhancing chaperone activity could suppress disease. However, bringing this to patients may not be so simple. Compounds that show some efficacy in mouse models are moving toward clinical trials, but whether they will enhance chaperone activity in the human brain is unknown.

Another potential approach to prevent disease is to impede the formation of putative toxic oligomers. If a compound can be developed that prevents expanded ataxin-3 and other polyQ disease proteins from aggregating, this may be beneficial. High throughput screens are underway in many laboratories to identify compounds that might act in this manner. Likewise, a compound that enhanced degradation of the mutant protein should effectively lower the concentration of abnormally folded protein and thereby reduce the rate of toxic aggregate formation.

In $\mathrm{HD}$ and certain SCAs, some evidence supports a role for mitochondrial impairment and increased oxidative stress as components to the pathogenic process. Accordingly, drugs to scavenge free radicals or to boost mitochondrial function are viable options for therapy. Along this line, the large clinical CARE-HD (Coenzyme Q10 and Remacemide Evaluation in HD) trial was conducted in symptomatic HD individuals. The results suggested a trend toward improvement, although this did not reach statistical significance. A second study employing higher doses of coenzyme Q10 in HD patients should begin soon. Unfortunately, although the $\mathrm{HD}$ field is leading the way in studying drugs to slow disease, the SCAs are lagging behind. Hopefully, when a positive result is found in HD trials it will spur investigators to try the same drug in a trial of MJD/SCA3 and other polyQ ataxias.

Finally, it may be possible to selectively turn off MJD1 and other SCA disease genes through the power of RNA interference. ${ }^{43}$ This has been accomplished in mouse models for certain polyQ diseases, including SCA1 and HD. ${ }^{44}$ Further preclinical studies are underway to test whether this can be brought to the clinic.

\section{AVAILABLE SYMPTOMATIC THERAPY SHOULD NOT BE FORGOTTEN}

The fact that MJD/SCA3 and all other SCAs are currently untreatable should not leave the physician with the notion that nothing can be done for affected persons. Nothing could be further from the truth. In $\mathrm{MJD} / \mathrm{SCA} 3$ and other SCAs, many symptoms that develop during the course of disease can be treated. Moreover, nonpharmacological measures can make a significant practical difference in quality of life.

Perhaps the clearest illustration of symptomatic therapy in MDJ/SCA3 is treatment of parkinsonism. ${ }^{14,15}$ Some patients develop features that closely resemble Parkinson's disease, including a pill-rolling rest tremor. These parkinsonian symptoms often respond quite well to dopamine replacement therapy, either with levodopa or a dopamine agonist. Some individuals with earlier onset dystonia and bradykinesia also may respond to dopaminergic therapy, although usually not as effectively as do those with more classic parkinsonian features.

Rapid eye movement (REM) sleep behavior disorder is common in MJD/SCA3. ${ }^{45,46}$ If obstructive sleep apnea is not a concern, treating REM sleep behavior disorder with clonazepam can help. I typically obtain a sleep study to rule out other sleep-related pathology before instituting benzodiazepines in patients with MJD/SCA3. Daytime sleepiness independent of nighttime sleep disturbance is also common. This may reflect destruction of brainstem nuclei implicated in levels of consciousness. In some patients, I have found that modafinil can be very effective in treating this. Similar sleep disturbances can also be seen in other SCAs.

RLS can be quite severe in MJD/SCA3 and, in some individuals with the smallest expansions, may be the only manifestation of disease. Again, dopaminergic therapy often works for this. Because peripheral neuropathy is a fairly frequent manifestation of MJD/SCA3, obtaining an electromyelogram with nerve conduction studies to rule out an additional component of neuropathic pain is 
appropriate. In addition, spasms and spasticity occur commonly in MJD/SCA3. Antispasmodics should be tried in anyone for whom spasms or spasticity is painful or disabling.

Of course, nonpharmacological interventions are just as important as pharmacological ones. Dysarthria and dysphagia affect nearly everyone with MJD/SCA3 and many other SCAs. A formal speech and swallowing evaluation by a therapist well versed in neurodegenerative diseases can provide patients with highly effective guidance in how and what to eat. This is as important for family members and caregivers as it is for the patient. Their own anxiety about the patient's mealtime can be alleviated by consulting with a speech and swallowing expert. As dysphagia worsens, follow-up oropharyngeal motility swallowing studies help to assess whether aspiration is significant enough to consider gastrostomy tube feedings.

One of the most significant complaints patients admit to is problems with vision. Often this takes the form of double vision due to impaired ocular motility. Prisms can help for those with relatively mild oculomotor problems.

Finally, physical therapy and occupational therapy assessments help considerably in deciding which gaitassist device is best for a patient at a given point in the disease, and in determining what additional measures need to be taken for the patient to continue employment or maintain independence in the home environment.

\section{UNDERSTANDING MACHADO-JOSEPH DISEASE/SPINOCEREBELLAR ATAXIA TYPE 3 PATHOGENESIS IS AIDED BY STUDIES OF RELATED DISORDERS}

A much-publicized pathological hallmark in polyQ diseases is the neuronal inclusion formed by the mutant protein. PolyQ inclusions are found in select brain regions, differing for each disease. Their discovery in MJD/ $\mathrm{SCA}^{47}$ and other polyQ diseases immediately suggested that protein misfolding might be central to pathogenesis. Although this view is now generally accepted by many researchers, it is important to recognize that the brain distribution of inclusions correlates imperfectly with regions of degeneration in MJD/SCA3 and other polyQ diseases, and in transgenic mouse models. Recent elegant studies in cell-based systems have dissociated inclusions from toxicity yet have also implied that misfolded, aggregated disease protein is intrinsically toxic. ${ }^{48,49}$ Based on studies from numerous polyQ diseases, the prevailing view is that inclusions in MJD/SCA3 and other polyQ SCAs represent, at minimum, biomarkers of a failure in mutant protein clearance and may even constitute a successful cellular response to protein aggregation.

Given the disease tissue evidence of aggregated protein, many investigators have studied the role of protein quality control components in polyQ diseases.
Together these studies have led to the view that various molecular chaperones, the ubiquitin-proteasome pathway, and the autophagosome/lysosome pathway constitute a key cellular defense against mutant ataxin-3 and other polyQ disease proteins. ${ }^{16,42}$ Neurons must ensure that proteins damaged by physiological stress or mutations are dealt with efficiently. If the concentration of misfolded proteins exceeds cellular folding and degradative capacity, these proteins can form insoluble aggregates that in turn become sequestered in inclusions. Because inclusions in $\mathrm{MJD} / \mathrm{SCA} 3$ and other polyQ disorders are ubiquitinated and sequester proteasome components, a failure in proteasomal degradation has been suggested to contribute to disease. ${ }^{27}$ To date, however, no one has shown in mouse models or in human disease tissue that polyQ protein directly inhibits the proteasome in neurons. One possibility is that impairment of protein quality control components, including the proteasome, is rather subtle and may be just one of several insults that neurons face when chronically exposed to expanded polyQ.

As in other SCAs, insights into MJD/SCA3 disease pathogenesis have stemmed from in vitro and cell-based experiments as well as from transgenic animal models. Of these, animal models may be the most revealing. For example, Bonini and colleagues have generated several Drosophila models of MJD/SCA3 that support a role for protein misfolding in pathogenesis. ${ }^{31,33}$ Studies in mice have also contributed substantially to our understanding of MJD/SCA3. For example, studies in the first mouse model of MJD/SCA3 showed that cerebellar expression of a polyQ carboxy-terminal fragment, but not of full-length ataxin-3, caused massive Purkinje cell degeneration and progressive ataxia. ${ }^{32}$ This result was the first to suggest that surrounding protein context greatly modulates polyQ toxicity, a common refrain now in studies of polyQ diseases. ${ }^{28}$

The study of MJD/SCA3 has recently been boosted by two new transgenic mouse models, both of which express full-length ataxin-3. One model expresses ataxin-3 from the full human MJD1 gene ${ }^{29}$ whereas the other model expresses ataxin-3 from a complementary DNA (cDNA) expressed behind the prion promoter. ${ }^{30}$ Both models display striking dose dependency: mice that are homozygous for the disease gene manifest much more severe disease than do hemizygous mice. Both models should prove valuable for exploring the role of neuronal dysfunction in disease, as the severe, behavioral phenotype observed in homozygous mice seems out of proportion to the relatively mild degree of brain atrophy in these mice. In other words, the disease phenotype probably reflects dysfunction of neurons more than it does cell death. In addition, the model expressing the full human MJD1 gene should allow scientists to assess the role of splice variants in disease and the relative regional brain expression of various isoforms of ataxin-3. ${ }^{50,51} \mathrm{In}$ 
many respects, this model also provides a closer approximation of the human disease in which to test various therapies, including RNA interference (RNAi).

With these mice in hand, researchers now can investigate pathogenic mechanisms in MJD, including the role of protein misfolding. Additional proposed pathogenic mechanisms for MJD/SCA3 and other polyQ diseases include the production of a toxic proteolytic fragment, transcriptional dysregulation, and perturbation of axonal transport.

With respect to the first of these, the toxic proteolytic fragment hypothesis, carboxy-terminal ataxin-3 fragments do accumulate in the cDNA mouse model. ${ }^{30}$ The protease responsible for this cleavage event has not been identified, but a similar ataxin- 3 fragment is generated by caspases in cells undergoing apoptosis. ${ }^{52}$ Moreover, caspases have been implicated in the generation of toxic fragments in many other polyQ diseases, most notably Huntington disease. As shown in MJD models in mice and flies, truncation of ataxin- 3 can accelerate aggregation and cytotoxicity, so proteolytic cleavage of ataxin- 3 could be important to the disease process.

Several cell-based and disease tissue-based studies are consistent with the hypothesis that expanded polyQ proteins trigger disease by perturbing gene transcription through aberrant protein-protein interactions. Although there is no reason to think ataxin-3 directly functions as a transcription factor, it does localize to nuclei in diseased neurons and has been shown to sequester the basal transcription factor TATA-binding protein (which is also the SCA17 disease protein) and the transcriptional coactivator CREB-binding protein into nuclear inclusions in cell-based models and in disease tissue. ${ }^{1}$ What has not been performed yet are gene expression analyses in the brain of mouse models of disease; these might provide new clues to pathogenic mechanisms in MJD/ SCA3. Finally, nontraditional roles for ubiquitin in nuclear events, including gene expression, are just now being uncovered. ${ }^{23}$ Thus, the deubiquitinating activity of ataxin-3, or its alteration in the disease state, could link directly to transcriptional regulation and deregulation.

The frequent occurrence of motor neuron loss and peripheral neuropathy in $\mathrm{MJD} / \mathrm{SCA} 3$ suggests that axonal dysfunction plays a significant role in disease. Aggregated disease proteins may physically block transport and titrate motor proteins away from their normal functions. Expression of a mutant ataxin-3 fragment in Drosophila, for example, was found to cause axonal blockages. ${ }^{24}$ In addition, ataxin-3 interacts with the cytosolic histone deacetylase 6 and dynein during transport of misfolded proteins in cultured cells, thus linking ataxin-3 to cytoskeletal transport processes. ${ }^{40}$ Despite these promising leads, the role of axonal pathology has not been studied rigorously in brains with MJD/SCA3. Clearly this aspect of disease warrants further study, both in human disease tissue and in transgenic mouse models.
In summary, although much has been ascertained about MJD/SCA3 pathogenesis from a wide variety of research endeavors, much more still needs to be learned before we have a clear understanding of the molecular events that cause neurons to become dysfunctional and ultimately degenerate in MJD/SCA3.

\section{ACKNOWLEDGMENTS}

The author's research on MJD/SCA3 is funded by grants from the National Institutes of Health, National Ataxia Foundation, and the Ataxia/MJD Research Project, Inc.

\section{REFERENCES}

1. Paulson H. Machado-Joseph disease/spinocerebellar ataxia type 3. In: Wells R, Ashizawa T, eds. Genetic Instabilities and Neurological Diseases. 2nd ed. San Diego: Elsevier/ Academic Press; 2005:363-377

2. Rub U, Brunt ER, de Vos RA, et al. Degeneration of the central vestibular system in spinocerebellar ataxia type 3 (SCA3) patients and its possible clinical significance. Neuropathol Appl Neurobiol 2004;30:402-414

3. Rub U, de Vos RA, Schultz C, et al. Spinocerebellar ataxia type 3 (Machado-Joseph disease): severe destruction of the lateral reticular nucleus. Brain 2002;125:2115-2124

4. Suenaga T, Matsushima H, Nakamura S, Akiguchi I, Kimura J. Ubiquitin-immunoreactive inclusions in anterior horn cells and hypoglossal neurons in a case with Joseph's disease. Acta Neuropathol (Berl) 1993;85:341-344

5. Murata Y, Yamaguchi S, Kawakami H, et al. Characteristic magnetic resonance imaging findings in Machado-Joseph disease. Arch Neurol 1998;55:33-37

6. Kawai Y, Takeda A, Abe Y, et al. Cognitive impairments in Machado-Joseph disease. Arch Neurol 2004;61:1757-1760

7. Kawaguchi Y, Okamoto T, Taniwaki M, et al. CAG expansions in a novel gene for Machado-Joseph disease at chromosome 14q32.1. Nat Genet 1994;8:221-228

8. Durr A, Stevanin G, Cancel G, et al. Spinocerebellar ataxia 3 and Machado-Joseph disease: clinical, molecular, and neuropathological features. Ann Neurol 1996;39:490-499

9. Cancel G, Abbas N, Stevanin G, et al. Marked phenotypic heterogeneity associated with expansion of a CAG repeat sequence at the spinocerebellar ataxia 3/Machado-Joseph disease locus. Am J Hum Genet 1995;57:809-816

10. Jardim LB, Pereira ML, Silveira I, et al. Neurologic findings in Machado-Joseph disease: relation with disease duration, subtypes, and (CAG)n. Arch Neurol 2001;58:899-904

11. Maciel P, Gaspar C, DeStefano AL, et al. Correlation between CAG repeat length and clinical features in Machado-Joseph disease. Am J Hum Genet 1995;57:54-61

12. Matilla T, McCall A, Subramony SH, Zoghbi HY. Molecular and clinical correlations in spinocerebellar ataxia type 3 and Machado-Joseph disease. Ann Neurol 1995;38: 68-72

13. Buhmann C, Bussopulos A, Oechsner M. Dopaminergic response in Parkinsonian phenotype of Machado-Joseph disease. Mov Disord 2003;18:219-221

14. Gwinn-Hardy K, Singleton A, O'Suilleabhain P, et al. Spinocerebellar ataxia type 3 phenotypically resembling Parkinson disease in a black family. Arch Neurol 2001;58:296-299 
15. Tuite PJ, Rogaeva EA, St George-Hyslop PH, Lang AE. Dopa-responsive parkinsonism phenotype of Machado-Joseph disease: confirmation of $14 \mathrm{q}$ CAG expansion. Ann Neurol 1995;38:684-687

16. Gatchel JR, Zoghbi HY. Diseases of unstable repeat expansion: mechanisms and common principles. Nat Rev Genet 2005;6:743-755

17. Moseley ML, Zu T, Ikeda Y, et al. Bidirectional expression of CUG and CAG expansion transcripts and intranuclear polyglutamine inclusions in spinocerebellar ataxia type 8 . Nat Genet 2006;38:758-769

18. Gu W, Ma H, Wang K, et al. The shortest expanded allele of the MJD1 gene in a Chinese MJD kindred with autonomic dysfunction. Eur Neurol 2004;52:107-111

19. Padiath QS, Srivastava AK, Roy S, Jain S, Brahmachari SK. Identification of a novel 45 repeat unstable allele associated with a disease phenotype at the MJD1/SCA3 locus. Am J Med Genet B Neuropsychiatr Genet 2005;133:124-126

20. van Alfen N, Sinke RJ, Zwarts MJ, et al. Intermediate CAG repeat lengths $(53,54)$ for $\mathrm{MJD} / \mathrm{SCA} 3$ are associated with an abnormal phenotype. Ann Neurol 2001;49:805-807

21. Takano H, Cancel G, Ikeuchi T, et al. Close associations between prevalences of dominantly inherited spinocerebellar ataxias with CAG-repeat expansions and frequencies of large normal CAG alleles in Japanese and Caucasian populations. Am J Hum Genet 1998;63:1060-1066

22. Schols L, Haan J, Riess O, Amoiridis G, Przuntek H. Sleep disturbance in spinocerebellar ataxias: is the SCA3 mutation a cause of restless legs syndrome? Neurology 1998;51:1603-1607

23. Berke SJ, Paulson HL. Protein aggregation and the ubiquitin proteasome pathway: gaining the upper hand on neurodegeneration. Curr Opin Genet Dev 2003;13:253-261

24. Gunawardena S, Goldstein LS. Polyglutamine diseases and transport problems: deadly traffic jams on neuronal highways. Arch Neurol 2005;62:46-51

25. Ross CA, Poirier MA. Protein aggregation and neurodegenerative disease. Nat Med 2004;10:S10-S17

26. Taylor JP, Hardy J, Fischbeck KH. Toxic proteins in neurodegenerative disease. Science 2002;296:1991-1995

27. Venkatraman P, Wetzel R, Tanaka M, Nukina N, Goldberg AL. Eukaryotic proteasomes cannot digest polyglutamine sequences and release them during degradation of polyglutamine-containing proteins. Mol Cell 2004;14:95-104

28. La Spada AR, Taylor JP. Polyglutamines placed into context. Neuron 2003;38:681-684

29. Cemal CK, Carroll CJ, Lawrence L, et al. YAC transgenic mice carrying pathological alleles of the MJD1 locus exhibit a mild and slowly progressive cerebellar deficit. Hum Mol Genet 2002;11:1075-1094

30. Goti D, Katzen SM, Mez J, et al. A mutant ataxin-3 putative-cleavage fragment in brains of Machado-Joseph disease patients and transgenic mice is cytotoxic above a critical concentration. J Neurosci 2004;24:10266-10279

31. Warrick JM, Morabito LM, Bilen J, et al. Ataxin-3 suppresses polyglutamine neurodegeneration in Drosophila by a ubiquitin-associated mechanism. Mol Cell 2005;18:37-48

32. Ikeda H, Yamaguchi M, Sugai S, et al. Expanded polyglutamine in the Machado-Joseph disease protein induces cell death in vitro and in vivo. Nat Genet 1996;13:196-202

33. Warrick JM, Paulson HL, Gray-Board GL, et al. Expanded polyglutamine protein forms nuclear inclusions and causes neural degeneration in Drosophila. Cell 1998;93:939949
34. Berke SJ, Chai Y, Marrs GL, Wen H, Paulson HL. Defining the role of ubiquitin-interacting motifs in the polyglutamine disease protein, ataxin-3. J Biol Chem 2005;280:32026-32034

35. Chai Y, Berke SS, Cohen RE, Paulson HL. Poly-ubiquitin binding by the polyglutamine disease protein ataxin-3 links its normal function to protein surveillance pathways. J Biol Chem 2004;279:3605-3611

36. Doss-Pepe EW, Stenroos ES, Johnson WG, Madura K. Ataxin-3 interactions with $\operatorname{rad} 23$ and valosin-containing protein and its associations with ubiquitin chains and the proteasome are consistent with a role in ubiquitin-mediated proteolysis. Mol Cell Biol 2003;23:6469-6483

37. Burnett B, Li F, Pittman RN. The polyglutamine neurodegenerative protein ataxin-3 binds polyubiquitylated proteins and has ubiquitin protease activity. Hum Mol Genet 2003;12:3195-3205

38. Mao Y, Senic-Matuglia F, Di Fiore PP, et al. Deubiquitinating function of ataxin-3: insights from the solution structure of the Josephin domain. Proc Natl Acad Sci USA 2005;102:12700-12705

39. Nicastro G, Menon RP, Masino L, et al. The solution structure of the Josephin domain of ataxin-3: structural determinants for molecular recognition. Proc Natl Acad Sci USA 2005;102:10493-10498

40. Burnett BG, Pittman RN. The polyglutamine neurodegenerative protein ataxin 3 regulates aggresome formation. Proc Natl Acad Sci USA 2005;102:4330-4335

41. Masino L, Musi V, Menon RP, et al. Domain architecture of the polyglutamine protein ataxin-3: a globular domain followed by a flexible tail. FEBS Lett 2003;549:21-25

42. Muchowski PJ, Wacker JL. Modulation of neurodegeneration by molecular chaperones. Nat Rev Neurosci 2005;6:11-22

43. Miller VM, Xia H, Marrs GL, et al. Allele-specific silencing of dominant disease genes. Proc Natl Acad Sci USA 2003;100:7195-7200

44. Xia H, Mao Q, Eliason SL, et al. RNAi suppresses polyglutamine-induced neurodegeneration in a model of spinocerebellar ataxia. Nat Med 2004;10:816-820

45. Friedman JH, Fernandez HH, Sudarsky LR. REM behavior disorder and excessive daytime somnolence in MachadoJoseph disease (SCA-3). Mov Disord 2003;18:1520-1522

46. Iranzo A, Munoz E, Santamaria J, et al. REM sleep behavior disorder and vocal cord paralysis in Machado-Joseph disease. Mov Disord 2003;18:1179-1183

47. Paulson HL, Perez MK, Trottier Y, et al. Intranuclear inclusions of expanded polyglutamine protein in spinocerebellar ataxia type 3. Neuron 1997;19:333-344

48. Arrasate M, Mitra S, Schweitzer ES, Segal MR, Finkbeiner S. Inclusion body formation reduces levels of mutant huntingtin and the risk of neuronal death. Nature 2004;431:805-810

49. Yang W, Dunlap JR, Andrews RB, Wetzel R. Aggregated polyglutamine peptides delivered to nuclei are toxic to mammalian cells. Hum Mol Genet 2002;11:2905-2917

50. Schmitt I, Evert BO, Khazneh H, Klockgether T, Wuellner U. The human MJD gene: genomic structure and functional characterization of the promoter region. Gene 2003;314:81-88

51. Schmidt T, Landwehrmeyer GB, Schmitt I, et al. An isoform of ataxin-3 accumulates in the nucleus of neuronal cells in affected brain regions of SCA3 patients. Brain Pathol 1998;8:669-679

52. Berke SJ, Schmied FA, Brunt ER, Ellerby LM, Paulson HL. Caspase-mediated proteolysis of the polyglutamine disease protein ataxin-3. J Neurochem 2004;89:908-918 